OPEN ACCESS

Edited by:

Marina A. Pavlova,

Eberhard Karls University of Tübingen,

Germany

Reviewed by:

Jan Van Den Stock,

Katholieke Universiteit Leuven,

Belgium

Michele Guerreschi,

Università di Brescia, Italy

${ }^{*}$ Correspondence:

Qin Zhang

zhangqin@cnu.edu.cn

Specialty section:

This article was submitted to

Emotion Science,

a section of the journal

Frontiers in Psychology

Received: 03 November 2015

Accepted: 02 June 2016

Published: 17 June 2016

Citation:

Wang Y and Zhang Q (2016)

Affective Priming by Simple Geometric Shapes: Evidence from

Event-related Brain Potentials.

Front. Psychol. 7:917.

doi: 10.3389/fpsyg.2016.00917

\section{Affective Priming by Simple Geometric Shapes: Evidence from Event-related Brain Potentials}

\author{
Yinan Wang and Qin Zhang* \\ Beijing Key Laboratory of Learning and Cognition, Department of Psychology, Capital Normal University, Beijing, China
}

Previous work has demonstrated that simple geometric shapes may convey emotional meaning using various experimental paradigms. However, whether affective meaning of simple geometric shapes can be automatically activated and influence the evaluations of subsequent stimulus is still unclear. Thus the present study employed an affective priming paradigm to investigate whether and how two geometric shapes (circle vs. downward triangle) impact on the affective processing of subsequently presented faces (Experiment 1) and words (Experiment 2). At behavioral level, no significant effect of affective congruency was found. However, ERP results in Experiment 1 and 2 showed a typical effect of affective congruency. The LPP elicited by affectively incongruent trials was larger compared to congruent trials. Our results provide support for the notion that downward triangle is perceived as negative and circle as positive and their emotional meaning can be activated automatically and then exert an influence on the electrophysiological processing of subsequent stimuli. The lack of significant congruent effect in behavioral measures and the inversed N400 congruent effect might reveal that the affective meaning of geometric shapes is weak because they are just abstract threatening cues rather than real threat. In addition, because no male participants are included in the present study, our findings are limited to females.

Keywords: circle, downward triangle, emotional meaning, affective priming paradigm, event-related potential (ERP), LPP

\section{INTRODUCTION}

Simple geometric patterns might convey emotional meaning. For example, diagonal and angular configurations tend to be associated with threat, while rounded features and curved lines tend to be linked to pleasantness and happiness. That association is evident in a wide array of observations, including facial features of tribal masks (Aronoff et al., 1988), preference for babyish faces and the caregiving for infants that may derive from their rounded facial features (Zebrowitz, 1997), and the configurations of human bodies in 17th-century Dutch art (Aronoff, 2006).

In addition to those observations, subjective evaluations in a few studies have demonstrated the emotional meaning of simple geometric shapes. Participants were asked to evaluate some geometric patterns on a set of subjective semantic differential scales (Osgood et al., 1957) and indicated the degree of "badness", "potency" and "activity" of each visual stimulus. It turned out that the sharp-v and rounded images conveyed an angry and a happy meaning, respectively (Aronoff et al., 1988, 1992). Bar and Neta (2006) obtained similar findings in a two-choice like/dislike forced choice task. Participants disliked neutral objects comprised of pointed features and sharp angles significantly 
more than curved ones (e.g., a watch with a sharp-angled contour in comparison with curved contour). Pavlova et al. (2005) also found a significant positive correlation between rated negative emotions and perceived instability of geometric shapes such as the triangle and the oval. Apart from those explicit subjective evaluations, Larson et al. (2012) used the Implicit Association Test to examine implicit attitudes toward circles and toward downward- and upward-pointing triangles. The results showed that participants were faster to categorize downward triangles as unpleasant, rather than neutral or pleasant, suggesting that people can extract affective meaning from simple geometric shapes and this affective perception could occur even without explicit affective value judgments.

Consistent with findings that stimuli that signal potential threat could capture attention preferentially, $\mathrm{v}$-shapes could be detected faster than some other shapes for their threat-related implications. Tipples et al. (2002) found faster detection of target faces (schematic face or the outline of a face) with threatening $\mathrm{v}$-shaped eyebrows compared to non-threatening $\Lambda$-shaped eyebrows, suggesting that a $\mathrm{v}$-shape played an important role in conveying negative meanings. Larson et al. (2007), using a visual search task, found that participants detected v-shapes or downward triangles faster than inverted $\mathrm{v}$-shapes or triangles, when those shapes were embedded in a field of other shapes. Moreover, Larson and colleagues demonstrated that, in some cases, during trials of homogeneous fields of stimuli, fields of $\mathrm{v}$-shapes led to slower response times, suggesting that this shape could cause difficulty in disengaging attention. Further evidence provided by Watson et al. (2011) showed that, when displays contained a varying number of elements and in which the stimuli were randomly arranged, searching for a downward triangle among upward triangles still was more efficient than the reverse. Furthermore, a search advantage for the downward-pointing triangle remained, regardless of whether the search display was presented within the context of a floor or ceiling perspective.

Processing of threatening stimuli such as v-shaped configuration or downward triangles also induced activation in the amygdala in several functional magnetic resonance imaging (fMRI) studies. For instance, the results of Bar and Neta (2007) revealed that neutral objects containing sharp features could activate the amygdala as compared to curved contours. Because previous studies (e.g., Vuilleumier et al., 2003; Whalen et al., 2004) showed that the amygdala exerted a particularly important influence on the detection of threat, the findings from Bar and Neta (2007) developed the idea further that sharp features can help signal a potential danger. Moreover, Larson et al. (2009) found that simple shapes with a downward $\mathrm{v}$ feature elicited greater activation of the amygdala and other neural networks (e.g., subgenual anterior cingulate cortex, fusiform gyrus, and superior temporal gyrus, and extrastriate visual regions). Those findings confirmed that simple v-shapes which were devoid of contextual affective cues could act like common emotional stimuli (such as facial expression) and activate the same neural circuitry known to process realistic, contextual threatening stimuli. Apart from fMRI evidence, recent research (Armbruster et al., 2014) investigated the effects of circles, as well as upwardand downward-point triangles, on three peripheral physiological markers: skin conductance response (SCR), startle reflex, and facial electromyography (EMG). In the first study, Armbruster et al. (2014) administered acoustic startle probes alone and during viewing of simple geometric shapes. In the second study, participants viewed the same geometric shapes, while measuring skin conductance on the non-dominant left hand and EMG over zygomaticus major and corrugators supercilii muscles. Armbruster et al. (2014) found that the presentation of circles resulted in significant reductions of startle and SCR magnitudes, compared to upward and downward pointing triangles, demonstrating that circle might be the most pleasant and least arousing among the three geometric shapes. In addition, Pavlova et al. (2010a) identified the cortical network engaged in visual processing of social interaction revealed by the motion of simple geometric shapes by analysis of oscillatory gamma magnetoencephalographic (MEG) activity. They found that the right posterior temporal cortex is a key region for the networks engaged in visual perception of social interaction. Moerover, a gender difference in the induced gamma response was found over the left prefrontal cortex, a perceptual decision-making region (Pavlova et al., 2010b).

It is worth noting that simple geometric shapes were taskrelevant stimuli in the above-mentioned experimental studies and in most of these studies participants were asked to explicitly respond to the shapes. What would happen, if these shapes are task-irrelevant stimuli and used only as backgrounds? Although many previous studies have explored emotional context effects (e.g., Van den Stock and de Gelder, 2014; Van den Stock et al., 2014a,b; Zhang et al., 2015), only a few studies have focused on effects of geometric shape context. In one study, Toet and Tak (2013) asked participants to judge perceived facial dominance of neutral faces presented on downward or upward background triangles. They found that participants judged neutral faces as more dominant on a downward triangle background, compared to an upward one. Other research (Watson et al., 2012) used the flanker task with simple geometric shapes as flanker stimuli. Participants evaluated the central face. The result showed that downward triangles interfered with valence judgments for the central target in a similar way to a negative face flanker: Responses were faster to negative face targets, but slower to positive ones, providing converging evidence that a downward triangle conveys negative valence.

The present study further examined effects of geometric shape context. In contrast to the studies of Watson et al. (2012) and Toet and Tak (2013), in which participants viewed the target stimulus and background shape simultaneously, the current study sequentially presented background and target to participants. More specifically, the present study employed the affective priming procedure in which participants viewed two affective stimuli in sequence and then evaluated the second stimulus (the target), without having to respond to the first one (the prime or context). The congruent effect occurs, when affectively congruent trials (i.e., a positive prime followed by a positive target or a negative prime followed by a negative target) lead to faster and less error-prone responses, compared to affectively incongruent trials (i.e., positive prime - negative target or negative prime - positive target). Because no response 
for primes was required, participants would process those shapes automatically. Thus, this procedure allows for investigation of whether the emotional meaning of different background shapes could be activated without active processing and then influence the participants' responses to targets. Our first experiment used downward triangles and circles as primes and used emotional faces as targets to investigate the effects of simple geometric forms on the evaluation of different emotional faces. In order to rule out the influence of the similarity between the circle and the facial contour on affective priming, the second experiment used the same prime stimuli, but with emotional words as targets. On the basis of substantive evidence from the literature that simple geometric shapes were associated with different emotional meanings, we expected that the affective meaning of these shapes could be automatically activated and then influence the evaluation of targets in a consistent manner. In other words, performance measured in terms of accuracy or reaction time (RT) would be better on congruent trials (downward trianglenegative target; circle-positive target) than on incongruent ones (downward triangle-positive target; circle-negative target).

In addition to behavioral measures, we also assessed event-related potential (ERP) measures to investigate the electrophysiological correlates of affective processing in the affective priming paradigm. Such electrophysiological indicators can reflect a temporally precise stream of neural activity from the moment a stimulus is presented until after the response is executed (Herring et al., 2011). We would concentrate on the N400 component and the late positive potential (LPP) based on previous affective priming studies (e.g., Werheid et al., 2005; Zhang et al., 2010, 2012). The N400 component is a negative deflection that generally peaks around $400 \mathrm{~ms}$ after target onset. Although many studies (see Kutas and Federmeier, 2011, for a recent review) have shown that its amplitude relies on the semantic relationship between the target and the context in which the target appears, N400 effects have also been found in the affective priming paradigm. Much research (e.g., Zhang et al., 2006, 2010; Krombholz et al., 2007; Eder et al., 2011) has demonstrated that affective mismatches between primes and targets evoked a larger N400 response than congruent pairs. LPP which appears in a time window between 400 and $700 \mathrm{~ms}$ is a positive component that is sensitive to the affective or motivational value of the stimuli. There is additional evidence in affective priming studies showing that evaluatively incongruent stimuli led to larger LPP amplitudes in comparison to evaluatively congruent ones, when participants categorize targets as positive or negative (Werheid et al., 2005; Zhang et al., 2010; Herring et al., 2011). In addition, we also examined the $\mathrm{N} 170$ component because different expressions would be used as targets in the present experiment. N170 is regarded as a face sensitive potential, which is indicated by a negative waveform in the $120-220 \mathrm{~ms}$ range with an average latency of $170 \mathrm{~ms}$ (Bentin et al., 1996). Many studies (e.g., Batty and Taylor, 2003; Eger et al., 2003; Blau et al., 2007; Leppänen et al., 2007; Japee et al., 2009) reported that facial emotional expression of real and morphed faces affected the face sensitive N170. For example, Hietanen and Astikainen (2013) recently showed a clear affective priming effect on this face sensitive component: N170 amplitudes to happy faces were larger when presented after positive than negative primes, whereas the N170 amplitudes to sad faces were larger when presented after negative than positive primes.

To sum up, the current study measured behavioral and electroencephalography (EEG) activity, applying an affective priming procedure that has been widely used as a tool to measure the effects of automatic evaluation, with downward triangles and circles as primes and expressions (Experiment 1) or words (Experiment 2) as targets. At behavioral level, we expected that affective priming should manifest as greater RTs and lower accuracy on affectively incongruent trials than on congruent trials. In electrophysiological terms, we focused our interest on the N170 at the P7 and P8 electrode sites (Experiment 1), as well as the N400 and LPP components on a wide range of brain areas (frontal, fronto-central, centro-parietal, and parietal) in both two experiments. According to the ERP studies mentioned above, we expected the N170 amplitudes to be larger for targets preceded by affectively congruent primes compared to incongruent primes. With respect to the N400 and LPP, we expected that the amplitudes would be greater in affectively incongruent primetarget trials than in affectively congruent prime-target trials. In addition, because previous studies (De Houwer et al., 2001; Werheid et al., 2005; Zhang et al., 2010; Hietanen and Astikainen, 2013; Okubo and Ogawa, 2013) have shown that the RT to positive facial expressions or words were significantly faster than to negative facial expressions or words in the affective priming procedure, we also manipulated target valence (positive vs. negative) to examine whether this factor could impact affective priming effect.

\section{MATERIALS AND METHODS}

\section{Participants}

Nineteen right-handed students (aged 19-24 years, mean age $=20.89$ years, $S D=1.41$ years) from Capital Normal University took part in two experiments. Only women were recruited in this study for ruling out the gender differences in emotion processing (Kring and Gordon, 1998; Kret and De Gelder, 2012). All participants had normal or correctedto-normal vision. Informed consent was obtained from each participant and all participants were financially compensated for their involvement. This study was approved by the Institutional Review Board of the Capital Normal University.

\section{Materials}

In each of two experiments, the stimuli consisted of 80 primetarget pairs, which were divided into two groups: 40 primetarget pairs using circles (o) as primes and 40 prime-target pairs using downward triangles $(\nabla)$ as primes. In addition, there were 40 filler pairs (squares as primes). Each of 120 pairs was repeatedly presented three times in each experiment. Assuming a viewing distance of $60 \mathrm{~cm}$, the visual angles were $3.82 \times 3.30^{\circ}$ for downward triangle, $3.82^{\circ}$ in diameter for circle, and $3.82 \times 3.82^{\circ}$ for squares. Another nineteen undergraduates who didn't participate in the two experiments evaluated each geometric shape on four 7-point scales (Lundqvist et al., 1999, 
2004; Lundqvist and Öhman, 2005) that were labeled from -3 (negative valence) to +3 (positive valence): Good-Bad, Kind-Cruel, Friendly-Unfriendly and Pleasant-Unpleasant. The valence of each shape was calculated as the mean of the four scales. The result showed that the average valence of the three shapes were $1.67,-0.61$, and 0.97 for circle, downward triangle, and square, respectively. The one-way repeated measures analysis of variance (ANOVA) revealed a main effect of valence, $F(2,56)=17.69, p<0.001$. Further analysis revealed that there were reliable differences on valence between circle and downward triangle $(p<0.001)$, as well as between square and downward triangle $(p<0.001)$. And the difference between circle and square reached a marginal significance $(p=0.081)$. These results indicated that circle was rated more positively than downward triangle on subjective judgments.

In Experiment 1, target stimuli were 40 pictures of faces (10 women and 10 men faces, 20 angry faces and 20 happy faces) selected from Chinese Facial Affective Picture System (Wang and Luo, 2005). The mean valence (a continuum ranging from unpleasant to pleasant) on a 1-9 point scale (with 9 being the most positive in valence dimension) was 2.88 for angry faces and 6.50 for happy faces, respectively. The mean arousal (a continuum ranging from calm to excited) on a 19 point scale (with 9 being the highest arousal) was 5.88 for angry faces and 5.12 for happy faces, respectively. There was no significant difference in arousal level between angry and happy faces $(p>0.05)$ but the valence difference between those two reached significance $(p<0.01)$. In order to make 80 primetarget pairs (40 affectively congruent pairs and 40 affectively incongruent pairs) and 40 filler pairs, each target was used in three separate pairs.

In Experiment 2, target stimuli were 20 negative words and 20 positive words which were chosen from the Chinese Affective Words System (Luo and Wang, 2004). The mean valence on a 1-9 point scale (with 9 being the most pleasant) was 2.98 for negative words and 6.70 for positive words, respectively. The mean familiarity (indicating how familiar people are with different words) on a 1-9 point scale (with 9 being the most familiar) was 5.41 for negative words and 5.34 for positive words, respectively. There was no significant difference in familiarity between negative and positive words $(p>0.05)$ but the valence difference between those two reached significance $(p<0.01)$.

\section{Procedure}

Stimuli were presented against a black background in the center of a $17^{\prime \prime}$ CRT monitor $(1024 \times 768$ resolution, $100-\mathrm{Hz}$ refresh rate). Each trial was started by a presentation of fixation cross "+" for $200 \mathrm{~ms}$ followed by a $300 \mathrm{~ms}$ blank screen (see Figure 1). Next, one of the three geometric shapes appeared for $100 \mathrm{~ms}$ followed by a blank screen ranging from 100 to $110 \mathrm{~ms}$ randomly. A target was then presented for $1500 \mathrm{~ms}$. Participants were instructed to categorize each face as angry or happy as quickly and accurately as possible in Experiment 1. And they were instructed to categorize each word as negative or positive in Experiment 2. Responses were entered via mouse key and the assignment of the left and right hand to target response was counterbalanced across

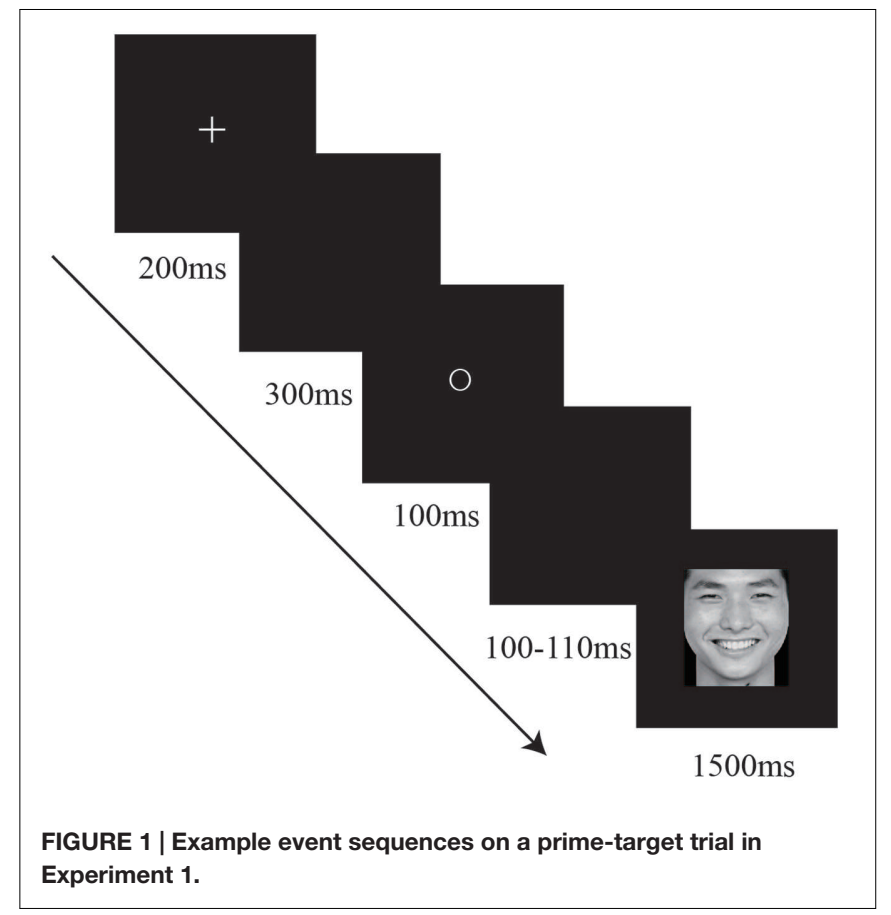

participants. Besides, each participant received three identical blocks in each experiment. The stimulus pairs were presented with random order in each block which consisted of 120 trials. Prior to testing, each participant performed 12 practice trials to ensure that the procedure was well understood.

\section{EEG Recordings and Data Processing}

Electroencephalogram (EEG) was continuously recorded from 64 scalp electrodes using an electrodes cap with $\mathrm{Ag} / \mathrm{AgCl}$ inserts. A left mastoid reference electrode was used online and the reference was changed offline to the average of left and right mastoid recordings. Vertical and horizontal electrooculograms (EOG) were recorded with two pairs of electrodes, one pair placed above and below the left eye, and another pair placed beside the two eyes. EEG signals were filtered with a band-pass from 0.05 to $40 \mathrm{~Hz}$ and sampled at a rate of $500 \mathrm{~Hz}$. Electrode impedances were kept below $5 \mathrm{~K} \Omega$. Average ERPs were formed offline from correctresponse trials. Epochs containing artifacts exceeding $\pm 75 \mu \mathrm{V}$ were excluded from ERP analyses. Each averaging epoch lasted $1200 \mathrm{~ms}$, including $400 \mathrm{~ms}$ prior to target onset.

The present study mainly focused on three ERP components: N170, N400 and LPP. The amplitude measurements were referred to pre-prime baseline ( -400 to $-210 \mathrm{~ms})$. Based on visual inspection of our waveform and a review of previous finding, mean amplitudes were calculated at time windows 130$210 \mathrm{~ms}$ (N170), 310-410 ms (N400), and 450-650 ms (LPP for Experiment 1) or 410-650 ms (LPP for Experiment 2). The N170 was measured at the P7 and P8 electrode sites and a 2(affective congruency: congruent, incongruent) $\times 2$ (target valence: angry, happy) $\times 2$ (electrode location: P7, P8) repeatedmeasures ANOVA was conducted for N170. Regarding N400 and LPP, the ANOVAs were conducted by selecting 24 electrodes 
from left hemisphere and right hemisphere at anterior locations (frontal and fronto-central) and posterior locations (centroparietal and parietal). These electrodes were divided into four areas: Left-Anterior (LA: F1, F3, F5, FC1, FC3, F5), Left-Posterior (LP: P1, P3, P5, CP1, CP3, CP5), Right-Anterior (RA: F2, F4, F6, FC2, FC4, FC6), and Right-Posterior (RP: P2, P4, P6, $\mathrm{CP} 2, \mathrm{CP} 4, \mathrm{CP} 6)$. The mean amplitude for each of the four areas under each condition was computed. Two congruency (congruent, incongruent) $\times 2$ target valence (angry/negative, happy/positive) $\times 2$ laterality (left, right) $\times 2$ location (anterior, posterior) repeated-measures ANOVAs were performed for N400 and LPP components.

\section{RESULTS}

Each participant's mean recognition accuracies and RTs to target faces (Experiment 1) or words (Experiment 2) were computed. Data beyond three standard deviations of the mean value were discarded from further analyses (2\% for both Experiments 1 and 2). The Shapiro-Wilk test for normality showed that RT data were normally distributed (Ws $>0.91, p s>0.05$ ).

\section{Experiment 1}

Table 1 displays mean RTs and accuracy rates under affectively congruent and incongruent conditions in Experiment 1.

A 2 (affective congruency: congruent, incongruent) $\times 2$ (target valence: angry, happy) repeated-measures ANOVA was performed on RTs. The main effect of congruency was not significant $\left[F(1,18)=0.19, p>0.05, \eta_{\mathrm{p}}^{2}=0.11\right]$. The main effect of target valence was significant $[F(1,18)=21.94, p<0.001$, $\left.\eta_{\mathrm{p}}^{2}=0.55,1-\beta=0.99\right]$, with slower responses when angry faces were presented compared to happy faces. The interaction between congruency and target valence also reached significance $\left[F(1,18)=7.47, p<0.05, \eta_{\mathrm{p}}^{2}=0.29,1-\beta=0.73\right]$ (see Figure 2). Simple effect analysis showed that participants responded to angry faces slower in congruent trials than incongruent trials $(p<0.05)$. Additionally, the two-way repeated-measures ANOVA for accuracies did not find any significant effects in Experiment 1 ( $p s>0.05)$.

In Experiment 1, the ERP data of eighteen participants were remained for analyses while one was excluded due to excessive ocular artifacts and electrode drift ( $>25 \%$ of trials). The ANOVA for N170 revealed a significant interaction between congruency and target valence $\left[F(1,17)=16.42, p<0.01, \eta_{\mathrm{p}}^{2}=0.49\right.$, $1-\beta=0.97]$. Simple effect analysis indicated that amplitudes for angry faces were more negative-going in incongruent trials than

TABLE 1 | Mean RTs (ms) and accuracies to angry and happy faces under different priming conditions ( $M \pm S D$ ) in Experiment 1.

\begin{tabular}{lccccc}
\hline & \multicolumn{2}{c}{ Angry faces } & & \multicolumn{2}{c}{ Happy faces } \\
\cline { 2 - 3 } \cline { 5 - 6 } & Incongruent & Congruent & & Incongruent & Congruent \\
\hline RT & $616 \pm 87$ & $626 \pm 88$ & & $597 \pm 93$ & $590 \pm 90$ \\
Accuracy & $0.97 \pm 0.03$ & $0.97 \pm 0.03$ & & $0.99 \pm 0.02$ & $0.98 \pm 0.02$
\end{tabular}

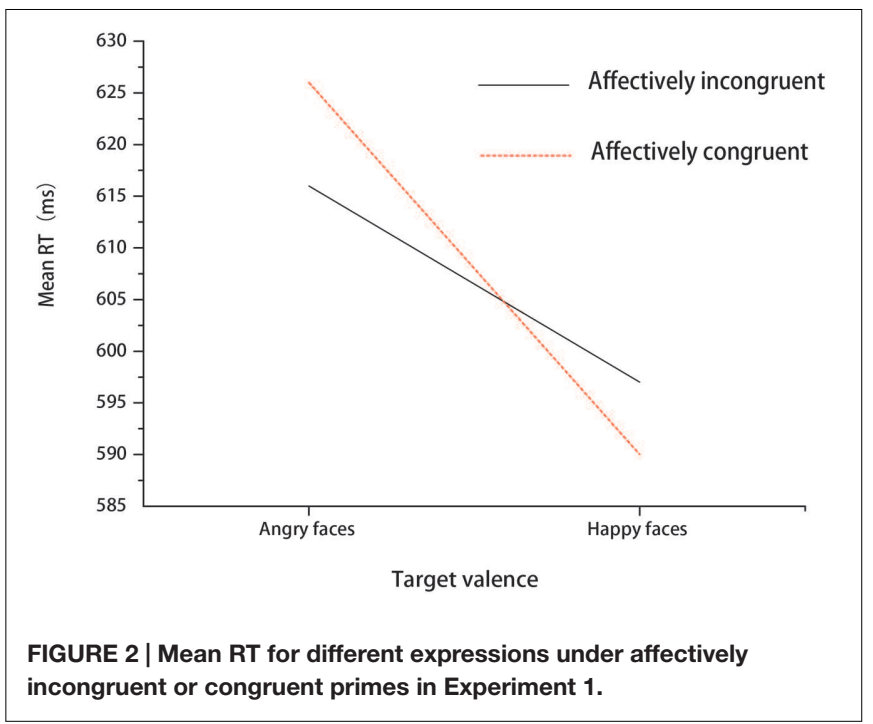

congruent trials $(p<0.001)$. On the contrary for happy faces, N170 amplitudes were more negative-going in congruent trials than incongruent trials $(p<0.001)$ (see Figure 3). No other main effects or interactions were significant $(p s>0.05)$. The analysis for N400 did not find any significant effects ( $p s>0.05)$. Regarding LPP, there was a significant main effect of location $\left[F(1,17)=6.56, p<0.05, \eta_{p}^{2}=0.28,1-\beta=0.68\right]$, with more positive-going amplitude on posterior areas compared to anterior areas. The main effect of target valence also reached significance $\left[F(1,17)=9.71, p<0.01, \eta_{\mathrm{p}}^{2}=0.63,1-\beta=0.84\right]$, due to more positive-going ERP amplitude for angry faces than happy faces. The interaction between location and target valence was significant $\left[F(1,17)=4.76, p<0.05, \eta_{\mathrm{p}}^{2}=0.22,1-\beta=0.54\right]$. No other main effects or interactions were significant $(p s>0.05)$. Then we performed an additional 2 congruency (congruent, incongruent) $\times 2$ target valence (angry, happy) repeatedmeasures ANOVA for LPP amplitude at the parietal-occipital site on midline $(\mathrm{POz})$ based on typical scalp distributions of LPP reported by previous studies (Lu et al., 2010; Herring et al., 2011) and our visual inspection of the grand average waveforms. There was a significant main effect of congruency $[F(1,17)=5.65$, $\left.p<0.05, \eta_{\mathrm{p}}^{2}=0.25,1-\beta=0.61\right]$, due to more positive-going ERP amplitude in incongruent trials than congruent trials (see Figure 4). A significant effect of target valence was also found $\left[F(1,17)=14.68, p<0.01, \eta_{\mathrm{p}}^{2}=0.46,1-\beta=0.95\right]$, with more positive-going ERP amplitude for angry faces than happy faces. The interaction between valence and target valance was not significant ( $p s>0.05)$.

\section{Experiment 2}

Table 2 displays mean RTs and accuracy rates under affectively congruent and incongruent conditions in Experiment 2.

A 2 (affective congruency: congruent, incongruent) $\times 2$ (target valence: negative, positive) repeated-measures ANOVA was performed on RTs. The main effect of congruency was not significant $\left[F(1,18)=1.64, p>0.05, \eta_{\mathrm{p}}^{2}=0.08\right]$. The main effect of target valence was significant $[F(1,18)=35.20, p<0.001$, 


\section{Angry face}
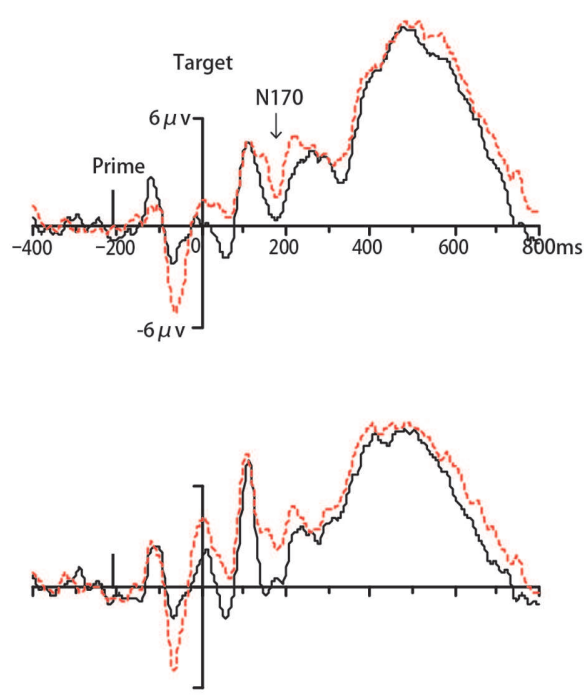

Affectively incongruent

\section{Happy face}

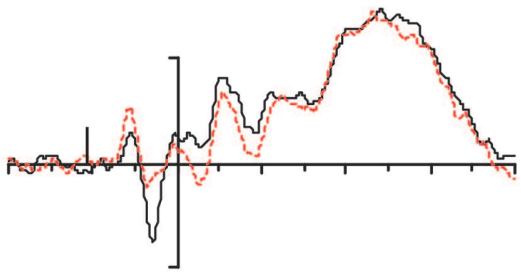

P8

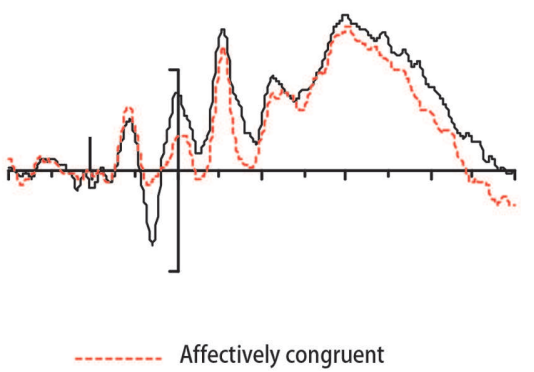

FIGURE 3 | Grand mean ERPs to different expressions from P7and P8 under affectively incongruent or congruent primes in Experiment 1.

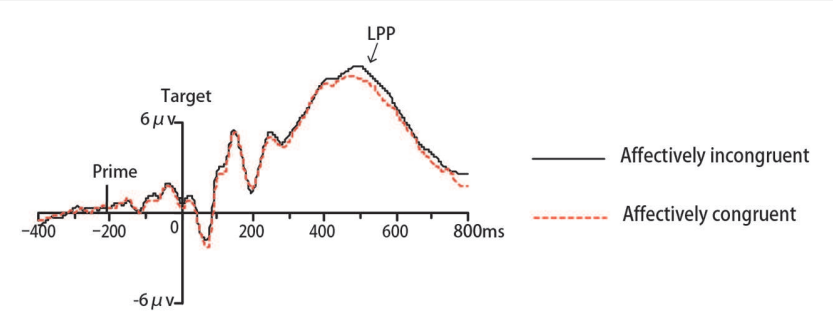

FIGURE 4 | Grand mean ERPs to expressions from POz under affectively incongruent or congruent primes in Experiment 1.

$\left.\eta_{\mathrm{p}}^{2}=0.66,1-\beta=1\right]$, with slower responses when negative words were presented compared to positive ones. The interaction between congruency and target valence did not reach significance $\left[F(1,18)=1.41, p>0.05, \eta_{\mathrm{p}}^{2}=0.29\right]$. Additionally, the twoway repeated-measures ANOVA for accuracies did not find any significant effects in Experiment 2 ( $p s>0.05)$.

The ERP data of seventeen participants were remained for analyses in Experiment 2 while two participants were excluded. The analysis for N400 indicated a significant main effect of congruency $\left[F(1,16)=4.64, p<0.05, \eta_{\mathrm{p}}^{2}=0.23,1-\beta=0.53\right]$. The average amplitude was significantly more negative-going to a target that was affectively congruent with the preceding prime compared to incongruent one (See Figure 5). The main effect of location also reached significance $[F(1,16)=8.82, p<0.05$, $\left.\eta_{\mathrm{p}}^{2}=0.36,1-\beta=0.80\right]$, with more negative-going amplitude on anterior areas compared to posterior areas. No other main effects or interactions were significant $(p s>0.05)$. The analysis for LPP indicated a significant effect of congruency $[F(1,16)=7.46$, $\left.p<0.05, \eta_{\mathrm{p}}^{2}=0.32,1-\beta=0.73\right]$, with incongruent trials elicited larger LPP amplitudes than congruent trials (See Figure 5). The main effect of location also reached significance $[F(1,16)=7.12$, $\left.p<0.05, \eta_{\mathrm{p}}^{2}=0.31,1-\beta=0.71\right]$, with more positive-going amplitude on posterior areas compared to anterior areas. The main effect of target valence was observed $[F(1,16)=6.72$, $\left.p<005, \eta_{p}^{2}=0.30,1-\beta=0.68\right]$, with more positive-going amplitudes elicited by negative words compared to positive words. No other main effects or interactions were significant $(p s>0.05)$

\section{DISCUSSION}

Previous work has demonstrated that simple geometric shapes may convey emotional meaning using various experimental paradigms. However, it has been unclear until now whether the emotional meaning of shapes could be activated without active processing and what the impact on the responses to a subsequent stimulus would be. Therefore, by employing an affective priming paradigm, we aimed to investigate whether the emotional meanings of circle and downward triangle could be

TABLE 2 | Mean RTs (ms) and accuracies to negative and positive words under different priming conditions $(M \pm S D)$ in Experiment 2.

\begin{tabular}{lccccc}
\hline & \multicolumn{2}{c}{ Negative words } & & \multicolumn{2}{c}{ Positive words } \\
\cline { 2 - 3 } \cline { 5 - 6 } & Incongruent & Congruent & & Incongruent & Congruent \\
\hline RT & $606 \pm 85$ & $616 \pm 96$ & & $581 \pm 95$ & $579 \pm 83$ \\
Accuracy & $0.97 \pm 0.04$ & $0.98 \pm 0.03$ & & $0.98 \pm 0.02$ & $0.97 \pm 0.04$
\end{tabular}


Left-Anterior

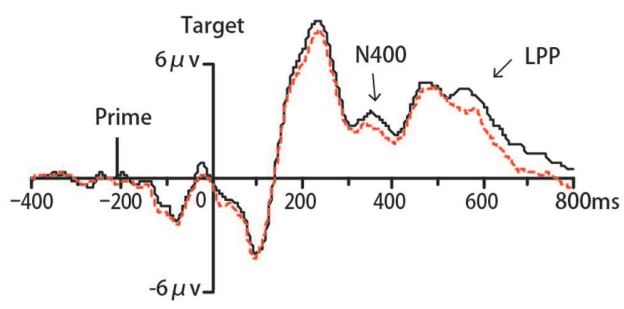

Left-Posterior

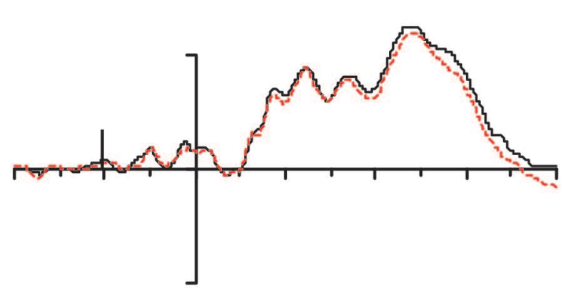

Affectively incongruent
Right-Anterior

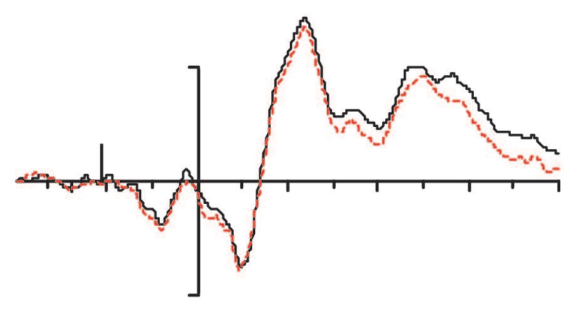

Right-Posterior

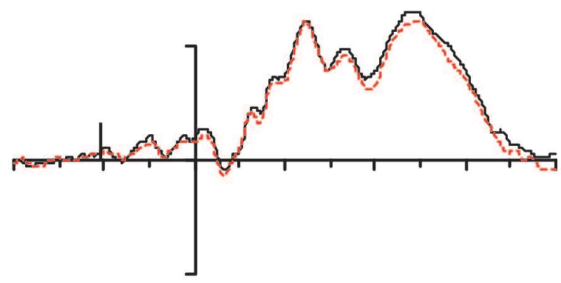

Affectively congruent

FIGURE 5 | Grand mean ERPs to words from four areas under affectively incongruent or congruent primes in Experiment 2.

activated automatically and then influence participants' responses to targets. We conducted two similar experiments, using same shapes (circle and downward triangle) as primes and either facial expressions (Experiment 1) or emotional words (Experiment 2) as targets.

The behavioral result of Experiment 1 showed a significant interaction between affective congruency and target valence. The responses to angry faces were faster after the presentation of incongruent primes than after congruent primes, which contradicted our expectation. We suppose that the structural similarity between circle and facial contour may have contributed to the experimental results. Previous work (Aronoff et al., 1988, 1992; Bar and Neta, 2006) and the subjective evaluation results of the present study all showed that the circle tends to be evaluated as positive, but the downward triangle tends to be evaluated as negative. Therefore, when angry faces were used as targets, prime stimuli were supposed to be circles in affectively incongruent trials and downward triangle in congruent trials according to those previous work and evaluation results. However, circle prime - angry face pair might be perceived as more congruent in structure than triangle prime - angry face pair. The structural similarity between the circle prime and the angry facial contour might have facilitated the responses to angry faces to some extent, resulting in faster evaluation in affectively incongruent trials than in congruent trials. When words were used as targets in the second experiment, the structural similarity between circle prime and target was ruled out. The unexpected congruency effect (i.e., faster responses in affectively incongruent trials than congruent ones) vanished. Additionally, the results of Experiment 1 did not indicate a facilitation effect of the circle on responses to happy faces. A possible explanation is that RTs to happy faces reached floor levels.

On the behavioral level, neither Experiment 1 nor 2 showed a significant affective priming effect in the expected direction. There might be two reasons for that outcome. On the one hand, the present evaluation task may not be sensitive enough to pick up subtle behavioral effects. In fact, such an affective congruency effect has not always been found in previous studies (Klauer and Musch, 2001; Storbeck and Robinson, 2004; Aguado et al., 2013) and several researchers have reported affective priming effects on the electrophysiological level in the absence of significant behavioral priming results (Hinojosa et al., 2009; Kissler and Koessler, 2011). On the other hand, compared to facial expressions or scenes with emotional meanings, simple geometric forms are more abstract and potentially weaker affective cues. When simple shapes were used as primes, the weak evaluative or response bias caused by them cannot effectively facilitate or inhibit the selection of the correct responses during the evaluation for targets. As a result, we did not observe a significant affective priming effect in the RT and accuracy rate data. That is, the affective meaning of simple geometric shapes may be not strong enough to influence the evaluation of the targets on a behavioral level. In fact, the subjective evaluation results showed that the downward triangle was only slightly negative, with a mean valence of -0.61 on a 7 -point scale from -3 (negative valence) to +3 (positive valence), supporting our explanation. Additionally, Armbruster et al. (2014) measured the influence of geometric shapes on peripheral physiological 
markers. Their results did not reveal a significant effect of geometric forms on facial EMG and there was also no significant difference between downward and upward triangles in SCR and startle magnitudes. Armbruster et al. (2014) concluded that, although the underlying neuronal pattern activated by downward triangles may be similar to that activated by real stimuli (such as angry faces), it was still necessary for individuals to differentiate between realistic threat stimuli and abstract geometric threat cues. This mechanism to discriminate was particularly important for individuals in order to facilitate appropriate responses and avoid unnecessary costly reactions (Armbruster et al., 2014). In the present study, the absence of a behavioral priming effect may reflect that participants were enabled to automatically differentiate between real affective stimuli and abstract affective cues (simple shapes).

In addition, our behavioral data showed that participants' responses to negative targets (angry expressions or negative words) were slower than those to positive targets (happy expressions or positive words). Such an effect had been reported in previous affective priming studies, either with expression targets (Werheid et al., 2005; Hietanen and Astikainen, 2013) or word targets (De Houwer et al., 2001; Zhang et al., 2010; Okubo and Ogawa, 2013). The effect probably suggests there is greater difficulty in disengaging attention from a negative stimulus than from a positive stimulus (Larson et al., 2007). Further investigations will be required to clarify this issue.

Compared to behavioral measures, ERP measurements can provide additional information. As we described in the Introduction, components of the ERP are known to be related to certain mental processes. The present study followed N170, N400, and LPP components with interest. According to our ERP data, the amplitudes of N170 showed opposite patterns during the evaluation of angry and happy faces, under two different priming conditions. More specifically, the N170 response to happy faces was more negative-going after affectively congruent primes (circles) than after incongruent primes (downward triangle), while the N170 elicited by angry faces was more positive-going after affectively congruent primes (downward triangle) than after affectively incongruent primes (circles). In other words, the $\mathrm{N} 170$ was more negative in the circle prime condition than in the downward triangle prime condition. Therefore, we thought that the present result reflected the effect of the priming type. In contrast with our results, Hietanen and Astikainen (2013), using an affective priming paradigm with natural scenes (prime) and expressions (target) as stimuli, found that the N170 amplitudes elicited by facial expressions were more negativegoing in affectively congruent trials than in incongruent trials. Hietanen and Astikainen (2013) argued that the N170 priming effect reflected the integration of activation elicited by the scenes and faces in specific brain regions. However, our Experiment 1 showed a priming type effect, but not an affectively congruent effect. Similar to the explanation of RT result, we think that the similarity between circle and face contour might play an important role in the present N170 effect. Considering that the N170 component reflects the visual processing of configurally represented information in the face (Bentin et al., 1996; Eimer, 2000; Sagiv and Bentin, 2001), it seems reasonable to suppose that it would be susceptible to the structural similarity with the stimuli used in the present study. Because the priming effect produced by this structural similarity might be stronger than the potential influence of affective congruency, the present study only showed a significant priming type effect.

The N400 component yielded a significant effect of affective congruency, but not in the expected direction, only in Experiment 2 using emotional words as targets. Affectively congruent trials yielded more negative-going N400, compared to incongruent ones. That outcome was contrary to many previous affective priming studies, which reported more negative-going N400 components in incongruent trials. Earlier language and memory studies (Kounios and Holcomb, 1992; Kutas and Federmeier, 2000; Federmeier, 2007) first examined the N400 component, as its amplitude relies on the semantic relationship between a target and the context before it appears. Subsequently, N400 has also been explored in a few affective priming studies, as it reflects the mismatch between a prime and a target. For instance, when Zhang et al. (2010) presented participants with pictures as primes and words as targets, the results showed an evaluation incongruity effect on the N400 component with a latency range of 350-450 ms. Zhang et al. (2010) found the N400 component to be more negative-going to affectively incongruent than to affectively congruent trials. Krombholz et al. (2007) also reported such an effect when participants were asked to evaluated expressions with words as primes. On the one hand, some studies have found enhanced N400 effects on incongruent trials (Morris et al., 2003; Steinbeis and Koelsch, 2009; Eder et al., 2011). On the other hand, inverse N400 priming effect has also been observed in some circumstances (i.e., enhanced N400 amplitude on congruent trials compared to incongruent ones) in previous studies (Herring et al., 2011; Kissler and Koessler, 2011). How might one explain these contradictory findings?

In semantic priming studies, the reversed effect on N400 component could be explained by a center-surround inhibition mechanism (Bermeitinger et al., 2008). According to that theory, in order to increase the activation of the prime concept, the concepts surrounding the prime would become inhibited. Thus, impeded access to related targets then evoked enhanced N400 (i.e., congruent trials with related targets showed more negativegoing amplitude). Consistent with the center-surround inhibition mechanism, Paulmann and Pell (2010) have reported a reversed N400 effect with faintly visible primes (SOA $=200 \mathrm{~ms}$ ). They supposed that faintly visible primes can only weakly activate the concepts associated with the prime. To increase the contrast between the prime concept and others, the surrounding concepts were inhibited, leading to more complex activities. Applying this explanation to the present study, we assumed that the brief presentation of a geometric form only weakly activated its corresponding emotional representation, inhibiting the processing of a related target. Therefore, this emotionally related target became less accessible compared to an unrelated one and gave rise to more negative-going amplitude on congruent trials, resulting in the inverse N400 priming effect we obtained. Of course, there might be other possible reasons leading to this reversed effect. Further studies are needed to resolve this 
question. Moreover, although the N400 effect occurred at all scalp locations, we found more negative-going amplitude at anterior locations than at posterior areas (see Figure 5). Such a frontal N400 was reported by Krombholz et al. (2007) using wordexpression pairs as stimuli. They inferred that the result reflected the involvement of frontal and prefrontal areas in cognitive and emotional processing.

Consistent with our expectation, a significant affective congruency effect on LPP occurred not only in Experiment1, but also in Experiment 2. Many studies (e.g., Werheid et al., 2005; Zhang et al., 2010, 2012; Herring et al., 2011; Hietanen and Astikainen, 2013) have demonstrated that the LPP component is modulated by affective congruency between the prime and the target. The affectively incongruent stimuli evoked larger amplitude LPP than affectively congruent ones and this LPP effect was considered as reflecting the increased attentional engagement under affectively incongruent conditions. Consistent with previous results, we also found a modulation of LPP by the affective congruency between the prime and the target, with enhanced LPP on incongruent trials compared to congruent ones. This result suggests that the emotional meaning of the circle and downward triangle can be activated automatically and can then influence the participants' evaluation of targets on an electrophysiological level. Specifically, when prime and target have opposite valence, participants must expend more effort to evaluate targets. However, one thing to note here is that we obtained a significant LPP effect only on the POz electrode, but not on a wide range of brain areas in Experiment 1. This might have resulted from the different types of targets. The cognitive mechanisms involved need to be explored further in future investigations. In addition, the present study showed that negative targets were associated with a more positive-going LPP than positive targets. That finding was consistent with several studies (e.g., Ito et al., 1998; Wood and Kisley, 2006) but inconsistent with other many studies (e.g., Schapkin et al., 2000; Delplanque et al., 2004; Herbert et al., 2008) which found enhanced LPP responses to pleasant compared to unpleasant stimuli. In the present study, we inferred that enhanced LPP responses to negative targets, together with slower RTs to negative targets, probably reflected more cognitive processing demands for negative stimuli and greater difficulty in disengaging attention from negative stimuli compared to positive stimuli.

There are several limitations to our studies. One is the lack of an appropriate neutral prime condition. Although we

\section{REFERENCES}

Aguado, L., Dieguez-Risco, T., Méndez-Bértolo, C., Pozo, M. A., and Hinojosa, J. A. (2013). Priming effects on the N400 in the affective priming paradigm with facial expressions of emotion. Cogn. Affect. Behav. Neurosci. 13, 284-296. doi: 10.3758/s13415-012-0137-3

Armbruster, D., Suchert, V., Gärtner, A., and Strobel, A. (2014). Threatening shapes: the impact of simple geometric configurations on peripheral physiological markers. Physiol. Behav. 135, 215-221. doi: 10.1016/j.physbeh.2014.06.020

Aronoff, J. (2006). How we recognize angry and happy emotion in people, places, and things. Cross Cult. Res. 40, 83-105. doi: 10.1177/10693971052 82597 included squares as shape primes in our experimental materials, the subjective evaluation results showed that the difference in valence between circle and square did not reach significance. Thus, squares are inadequate as neutral shape primes. Future research with demonstrably neutral prime stimuli is needed to help determine the source of affective congruency effects. Another limitation of the present study is a relative small sample size. Although our experiments had sufficient power to detect the influences of simple shapes, based on $p$ values and effect size data provided in the result section, future studies with larger sample sizes would help support our conclusions. Finally, because no male participants took part in our experiments, our results are limited to female participants and the gender differences on the evaluation of simple geometric shapes cannot be investigated as well. Previous studies have showed some indications for gender differences in social cognition (see Pavlova, 2012). Further research including female and male participants is needed to explore the gender effect on the evaluation of shapes.

To sum up, using an affective priming paradigm, two experiments examined whether and how a circle and a downward triangle influence the affective processing of the faces and words. Although the expected behavioral effect of affective congruency did not emerge, we did find the hypothesized congruency effects at the electrophysiological level, mainly manifest as larger LPP component change in affectively incongruent trials compared to congruent trials. This LPP priming effect might suggest that the emotional meaning of a circle and a downward triangle is activated automatically and then impacts on the electrophysiological processing of subsequent stimuli. However, our findings are limited to females.

\section{AUTHOR CONTRIBUTOINS}

YW co-designed the experiment, collected, and analyzed the data and co-wrote the text. QZ co-designed the experiment, advised on many aspects of the research and co-wrote the text.

\section{ACKNOWLEDGMENTS}

This research was supported by the National Natural Science Foundation of China (31470980).

Aronoff, J., Barclay, A. M., and Stevenson, L. A. (1988). The recognition of threatening facial stimuli. J. Pers. Soc. Psychol. 54, 647-655. doi: 10.1037/00223514.54.4.647

Aronoff, J., Woike, B. A., and Hyman, L. M. (1992). Which are the stimuli in facial displays of anger and happiness? Configurational bases of emotion recognition. J. Pers. Soc. Psychol. 62, 1050-1066. doi: 10.1037/0022-3514.62.6.1050

Bar, M., and Neta, M. (2006). Humans prefer curved visual objects. Psychol. Sci. 17, 645-648. doi: 10.1111/j.1467-9280.2006.01759.x

Bar, M., and Neta, M. (2007). Visual elements of subjective preference modulate amygdala activation. Neuropsychologia 45, 2191-2200. doi: 10.1016/j.neuropsychologia.2007.03.008

Batty, M., and Taylor, M. J. (2003). Early processing of the six basic facial emotional expressions. Cogn. Brain Res. 17, 613-620. doi: 10.1016/S0926-6410(03)00174-5 
Bentin, S., Allison, T., Puce, A., Perez, E., and McCarthy, G. (1996). Electrophysiological studies of face perception in humans. J. Cogn. Neurosci. 8, 551-565. doi: 10.1162/jocn.1996.8.6.551

Bermeitinger, C., Frings, C., and Wentura, D. (2008). Reversing the N400: Eventrelated potentials of a negative semantic priming effect. NeuroReport 19, 1479-1482. doi: 10.1097/WNR.0b013e32830f4b0b

Blau, V. C., Maurer, U., Tottenham, N., and McCandliss, B. D. (2007). The facespecific N170 component is modulated by emotional facial expression. Behav. Brain Funct. 3:7. doi: 10.1186/1744-9081-3-7

De Houwer, J., Hermans, D., and Spruyt, A. (2001). Affective priming of pronunciation responses: effects of target degradation. J. Exp. Soc. Psychol. 37:85. doi: $10.1006 /$ jesp.2000.1437

Delplanque, S., Lavoie, M. E., Hot, P., Silvert, L., and Sequeira, H. (2004). Modulation of cognitive processing by emotional valence studied through event-related potentials in humans. Neurosci. Lett. 356, 1-4. doi: 10.1016/j.neulet.2003.10.014

Eder, A. N., Leuthold, H., Rothermund, K., and Schweinberger, S. R. (2011). Automatic response activation in sequential affective priming: an ERP study. Soc. Cogn. Affect. Neurosci. 7, 436-445. doi: 10.1093/scan/nsr033

Eger, E., Jedynak, A., Iwaki, T., and Skrandies, W. (2003). Rapid extraction of emotional expression: evidence from evoked potential fields during brief presentation of face stimuli. Neuropsychologia 41, 808-817. doi: 10.1016/S00283932(02)00287-7

Eimer, M. (2000). The face-specific N170 component reflects late stages in the structural encoding of faces. Neuroreport 11, 2319-2324. doi: 10.1097/00001756-200007140-00050

Federmeier, K. D. (2007). Thinking ahead: the role and roots of prediction in language comprehension. Psychophysiology 44, 491-505. doi: 10.1111/j.14698986.2007.00531.x

Herbert, C., Junghofer, M., and Kissler, J. (2008). Event related potentials to emotional adjectives during reading. Psychophysiology 45, 487-498. doi: 10.1111/j.1469-8986.2007.00638.x

Herring, D. R., Taylor, J. H., White, K. R., and Crites, S. J. (2011). Electrophysiological responses to evaluative priming: the LPP is sensitive to incongruity. Emotion 11, 794-806. doi: 10.1037/a00 22804

Hietanen, J. K., and Astikainen, P. (2013). N170 response to facial expressions is modulated by the affective congruency between the emotional expression and preceding affective picture. Biol. Psychol. 92, 114-124. doi: 10.1016/j.biopsycho.2012.10.005

Hinojosa, J. A., Carretié, L., Méndez-Bértolo, C., Míguez, A., and Pozo, M. A. (2009). Arousal contributions to affective priming: electrophysiological correlates. Emotion 9, 164-171. doi: 10.1037/a0014680

Ito, T. A., Larsen, J. T., Smith, N. K., and Cacioppo, J. T. (1998). Negative information weighs more heavily on the brain: the negativity bias in evaluative categorizations. J. Pers. Soc. Psychol. 75, 887-900. doi: 10.1037/00223514.75.4.887

Japee, S., Crocker, L., Carver, F., Pessoa, L., and Ungerleider, L. G. (2009). Individual differences in valence modulation of face-selective $\mathrm{m} 170$ response. Emotion 9, 59-69. doi: 10.1037/a0014487

Kissler, J., and Koessler, S. (2011). Emotionally positive stimuli facilitate lexical decisions-An ERP study. Biol. Psychol. 86, 254-264. doi: 10.1016/j.biopsycho.2010.12.006

Klauer, K. C., and Musch, J. (2001). Does sunshine prime loyal? Affective priming in the naming task. Q. J. Exp. Psychol. A 54, 727-751. doi: $10.1080 / 02724980042000444$

Kounios, J., and Holcomb, P. J. (1992). Structure and process in semantic memory: evidence from event-related brain potentials and reaction times. J. Exp. Psychol. Gen. 121, 459-479. doi: 10.1037/0096-3445.121.4.459

Kret, M. E., and De Gelder, B. (2012). A review on sex differences in processing emotional signals. Neuropsychologia 50, 1211-1221. doi: 10.1016/j.neuropsychologia.2011.12.022

Kring, A. M., and Gordon, A. H. (1998). Sex differences in emotion: expression, experience, and physiology. J. Pers. Soc. Psychol. 74, 686-703. doi: 10.1037/00223514.74.3.686

Krombholz, A., Schaefer, F., and Boucsein, W. (2007). Modification of N170 by different emotional expression of schematic faces. Biol. Psychol. 76, 156-162. doi: 10.1016/j.biopsycho.2007.07.004
Kutas, M., and Federmeier, K. D. (2000). Electrophysiology reveals semantic memory use in language comprehension. Trends Cogn. Sci. 4, 463-470. doi: 10.1016/S1364-6613(00)01560-6

Kutas, M., and Federmeier, K. D. (2011). Thirty years and counting: finding meaning in the N400 component of the event-related brain potential (ERP). Annu. Rev. Psychol. 62, 621-647. doi: 10.1146/annurev.psych.093008.131123

Larson, C. L., Aronoff, J., Sarinopoulos, I. C., and Zhu, D. C. (2009). Recognizing threat: simple geometric shapes activate neural circuitry underlying threat detection. J. Cogn. Neurosci. 21, 1523-1535. doi: 10.1162/jocn.2009.21111

Larson, C. L., Aronoff, J., and Stearns, J. J. (2007). The shape of threat: simple geometric forms evoke rapid and sustained capture of attention. Emotion 7, 526-534. doi: 10.1037/1528-3542.7.3.526

Larson, C. L., Aronoff, J., and Steuer, E. L. (2012). Simple geometric shapes are implicitly associated with affective value. Motiv. Emot. 36, 404-413. doi: 10.1007/s11031-011-9249-2

Leppänen, J. M., Kauppinen, P., Peltola, M. J., and Hietanen, J. K. (2007). Differential electrocortical responses to increasing intensities of fearful and happy emotional expressions. Brain Res. 1166, 103-109. doi: 10.1016/j.brainres.2007.06.060

Lu, Y., Zhang, W. N., and Shen, D. L. (2010). Subliminal affective priming effect by faces with different valence: an ERP study. Acta Psychol. Sin. 42, 929-938. doi: 10.3724/SP.J.1041.2010.00929

Lundqvist, D., Esteves, F., and Ohman, A. (1999). The face of wrath: critical features for conveying facial threat. Cogn. Emot. 13, 691-711. doi: 10.1080/026999399379041

Lundqvist, D., Esteves, F., and Öhman, A. (2004). The face of wrath: the role of features and configurations in conveying social threat. Cogn. Emot. 18, 161-182. doi: $10.1080 / 02699930244000453$

Lundqvist, D., and Öhman, A. (2005). Emotion regulates attention: the relation between facial configurations, facial emotion, and visual attention. Vis. Cogn. 12, 51-84. doi: 10.1080/13506280444000085

Luo, Y. J., and Wang, Y. N. (2004). Chinese Affective Words System (CAWS). Beijing: Institute of Psychology, CAS.

Morris, J. P., Squires, N. K., Taber, C. S., and Lodge, M. (2003). Activation of political attitudes: a psychophysiological examination of the hot cognition hypothesis. Polit. Psychol. 24:727. doi: 10.1046/j.1467-9221.2003.00349.x

Okubo, C., and Ogawa, T. (2013). Unconscious and conscious processing of negative emotions examined through affective priming. Psychol. Rep. 112, 607-625. doi: 10.2466/21.07.PR0.112.2.607-625

Osgood, C. E., Suci, G. J., and Tannenbaum, P. H. (1957). The Measurement of Meaning. Urbana, IL: University of Illinois Press.

Paulmann, S., and Pell, M. D. (2010). Contextual influences of emotional speech prosody on face processing: how much is enough? Cogn. Affect. Behav. Neurosci. 10, 230-242. doi: 10.3758/CABN.10.2.230

Pavlova, M., Guerreschi, M., Lutzenberger, W., and Krägeloh-Mann, I. (2010a). Social interaction revealed by motion: dynamics of neuromagnetic gamma activity. Cereb. Cortex 20, 2361-2367. doi: 10.1093/cercor/bhp304

Pavlova, M., Guerreschi, M., Lutzenberger, W., Sokolov, A. N., and KrägelohMann, I. (2010b). Cortical response to social interaction is affected by gender. Neuroimage 50, 1327-1332. doi: 10.1016/j.neuroimage.2009.12.096

Pavlova, M., Sokolov, A. A., and Sokolov, A. (2005). Perceived dynamics of static images enables emotional attribution. Perception 34, 1107-1116. doi: $10.1068 /$ p5 500

Pavlova, M. A. (2012). Biological motion processing as a hallmark of social cognition. Cereb. Cortex 22, 981-995. doi: 10.1093/cercor/bhr156

Sagiv, N., and Bentin, S. (2001). Structural encoding of human and schematic faces: holistic and part-based processes. J. Cogn. Neurosci. 13, 937-951. doi: $10.1162 / 089892901753165854$

Schapkin, S. A., Gusev, A. N., and Kuhl, J. (2000). Categorization of unilaterally presented emotional words: an ERP analysis. Acta Neurobiol. Exp. 60, 17-28.

Steinbeis, N., and Koelsch, S. (2009). Understanding the intentions behind man-made products elicits neural activity in areas dedicated to mental state attribution. Cereb. Cortex 19, 619-623. doi: 10.1093/cercor/bhn110

Storbeck, J., and Robinson, M. D. (2004). Preferences and inferences in encoding visual objects: a systematic comparison of semantic and affective priming. Pers. Soc. Psychol. Bull. 30, 81-93. doi: 10.1177/0146167203258855

Tipples, J., Atkinson, A. P., and Young, A. W. (2002). The eyebrow frown: a salient social signal. Emotion 2, 288-296. doi: 10.1037/1528-3542.2.3.288 
Toet, A., and Tak, S. (2013). Look out, there is a triangle behind you! The effect of primitive geometric shapes on perceived facial dominance. Iperception 4, 53-56. doi: 10.1068/i0568sas

Van den Stock, J., and de Gelder, B. (2014). Face identity matching is influenced by emotions conveyed by face and body. Front. Hum. Neurosci. 8:53. doi: 10.3389/fnhum.2014.00053

Van den Stock, J., Vandenbulcke, M., Sinke, C. B., and de Gelder, B. (2014a). Affective scenes influence fear perception of individual body expressions. Hum. Brain Mapp. 35, 492-502. doi: 10.1002/hbm.22195

Van den Stock, J., Vandenbulcke, M., Sinke, C. B., Goebel, R., and de Gelder, B. (2014b). How affective information from faces and scenes interacts in the brain. Soc. Cogn. Affect. Neurosci. 9, 1481-1488. doi: 10.1093/scan/nst138

Vuilleumier, P., Armony, J. L., Driver, J., and Dolan, R. J. (2003). Distinct spatial frequency sensitivities for processing faces and emotional expressions. Nat. Neurosci. 6, 624-631. doi: 10.1038/nn1057

Wang, Y., and Luo, Y. J. (2005). Standardization and assessment of college students' facial expression of emotion. Chin. J. Clini. Psychol. 13, 396-398.

Watson, D. G., Blagrove, E., Evans, C., and Moore, L. (2012). Negative triangles: simple geometric shapes convey emotional valence. Emotion 12, 18-22. doi: 10.1037/a0024495

Watson, D. G., Blagrove, E., and Selwood, S. (2011). Emotional triangles: a test of emotion-based attentional capture by simple geometric shapes. Cogn. Emot. 25, 1149-1164. doi: 10.1080/02699931.2010.525861

Werheid, K., Alpay, G., Jentzsch, I., and Sommer, W. (2005). Priming emotional facial expressions as evidenced by event-related brain potentials. Int. J. Psychophysiol. 55, 209-219. doi: 10.1016/j.ijpsycho.2004.07.006

Whalen, P. J., Kagan, J., Cook, R. G., Davis, F. C., Kim, H., Polis, S., et al. (2004). Human amygdala responsivity to masked fearful eye whites. Science 306:2061. doi: $10.1126 /$ science. 1103617
Wood, S., and Kisley, M. A. (2006). The negativity bias is eliminated in older adults: age-related reduction in event-related brain potentials associated with evaluative categorization. Psychol. Aging 21, 815-820. doi: 10.1037/08827974.21.4.815

Zebrowitz, L. A. (1997). Reading Faces. Boulder, CO: Westview Press.

Zhang, Q., Kong, L., and Jiang, Y. (2012). The interaction of arousal and valence in affective priming: behavioral and electrophysiological evidence. Brain Res. 1474, 60-72. doi: 10.1016/j.brainres.2012.07.023

Zhang, Q., Lawson, A., Guo, C., and Jiang, Y. (2006). Electrophysiological correlates of visual affective priming. Brain Res. Bull. 71, 316-323. doi: 10.1016/j.brainresbull.2006.09.023

Zhang, Q., Li, X., Gold, B. T., and Jiang, Y. (2010). Neural correlates of cross-domain affective priming. Brain Res. 1329, 142-151. doi: 10.1016/j.brainres.2010.03.021

Zhang, Q., Liu, X., An, W., Yang, Y., and Wang, Y. (2015). Recognition memory of neutral words can be impaired by task-irrelevant emotional encoding contexts: behavioral and electrophysiological evidence. Front. Hum. Neurosci. 9:73. doi: 10.3389/fnhum.2015.00073

Conflict of Interest Statement: The authors declare that the research was conducted in the absence of any commercial or financial relationships that could be construed as a potential conflict of interest.

Copyright (c) 2016 Wang and Zhang. This is an open-access article distributed under the terms of the Creative Commons Attribution License (CC BY). The use, distribution or reproduction in other forums is permitted, provided the original author(s) or licensor are credited and that the original publication in this journal is cited, in accordance with accepted academic practice. No use, distribution or reproduction is permitted which does not comply with these terms. 